\title{
Study of Aoki phase in Nc=2 gauge theories with fundamental and adjoint fermions
}

\author{
Hideo Matsufuru* \\ High Energy Accelerator Research Organization (KEK), Tsukuba 305-0801, Japan \\ E-mail: hideo.matsufuru@kek.jp \\ Yoshio Kikukawa \\ Institute of Physics, University of Tokyo, Tokyo 153-8092, Japan \\ E-mail: kikukawa@hep1.c.u-tokyo.ac.jp \\ Norikazu Yamada \\ KEK Theory Center, Institute of Particle and Nuclear Studies, High Energy Accelerator \\ Research Organization (KEK), Tsukuba 305-0801, Japan, \\ and School of High Energy Accelerator Science, The Graduate University for Advanced Studies \\ (Sokendai), Tsukuba 305-0801, Japan \\ E-mail: norikazu.yamada@kek.jp
}

\begin{abstract}
We study the phase structure of the $N_{c}=2$ lattice gauge theories with fundamental and adjoint fermion representations in the supercritical (negative mass) region of the Wilson-Dirac operator. To examine the location of the Aoki phase, we investigate the behaviors of meson correlation functions as well as the locality of the low-lying eigenmodes of the hermitian Wilson-Dirac operator, on quenched and dynamical configurations. We also observe the level statistics of the overlap operator and compare them with the random matrix theory.
\end{abstract}

The XXVII International Symposium on Lattice Field Theory

July 26-31, 2009

Peking University, Beijing, China

${ }^{*}$ Speaker. 


\section{Introduction}

Recently it has been drawing much attention to study non-QCD lattice gauge theories with general numbers of colors and flavors and with general fermion representations [1]. Main motivation is a search for possible alternative dynamics to the Higgs sector in the standard model, whose effect may become manifest in forthcoming LHC experiment. For instance, in the so-called walking technicolor theories, a slowly running (walking) coupling over wide range of scale plays an essential role, and gauge theories with a nontrivial infrared fixed point and a conformal window are regarded as possible candidates with such dynamical property. Lattice simulations now enable us to study these candidate gauge theories, and to estimate physical quantities to be compared with current and future precision experimental data.

Our goal of present study is to explore the $N_{c}=2$ lattice gauge theories with $N_{f}$ massless Dirac fermions in fundamental or adjoint representation, and examine the dynamics of spontaneous chiral symmetry breaking for various $N_{f}$. By adopting the overlap-Dirac fermions, which have an exact chiral symmetry on the lattice, one can simulate the theories in the $\varepsilon$-regime and examine possible chiral symmetry breaking through the low-lying spectrum of the overlap-Dirac operator. The relation of chiral phase transition to deconfinement transition in these theories is another interesting subject to study. These $\mathrm{SU}(2)$ gauge theories have an attractive feature that the infrared fixed point is expected to appear with less number of $N_{f}$, compared to larger $N_{c}$ theories.

In using the overlap fermions, one needs to ensure that the overlap-Dirac operator is exponentially local. Since the locality of the overlap operator is closely related to the spectral gap of the Wilson-Dirac operator with a supercritical mass, we first focus on this issue in $N_{c}=2$ case. Namely, we explore the Aoki phase, where the flavor-parity symmetry is broken spontaneously (in addition to chiral symmetry), by analyzing the meson correlator and the spectrum of the Wilson-Dirac operator. We also investigate the spectrum of the overlap operator to compare with the random matrix theory. All results in this report are obtained in quenched approximation, as a preparation for the dynamical simulations of overlap fermions.

\section{Locality of overlap operator and Aoki phase}

The overlap-Dirac operator is represented as

$$
D_{o v}=\frac{M_{0}}{a}\left[1+\gamma_{5} \operatorname{sign}\left(H_{W}\left(-M_{0}\right)\right)\right],
$$

where $H_{W}$ is the hermitian Wilson-Dirac operator with negative mass $-M_{0}$. If the near-zero eigenvalue spectrum of $H_{W}$ has a gap, the locality of the overlap operator is ensured [2]. In the case that the near-zero modes exist, Golterman and Shamir proposed the following conjecture $[3,4]$.

- There is certain frequency below that the eigenvectors of $H_{W}$ are exponentially localized. This frequency is called 'mobility edge' after similar phenomena in the disordered system.

- If the near-zero modes of $H_{W}$ is exponentially local, the overlap operator is also local.

- Aoki phase is characterized with vanishing mobility edge. 
This conjecture is numerically confirmed in Refs. [4]. The locality of the overlap operator is also verified along this conjecture in Refs. [5]. Thus the locality of the overlap operator is satisfied if the system is out of the Aoki phase.

The Aoki phase is characterized by parity-flavor symmetry breaking [6]. Let us consider the $N_{f}=2$ QCD case. At zero temperature, the chiral symmetry is spontaneously broken, and three massless Nambu-Goldstone modes (pions) appear accordingly. For the Wilson-Dirac operator with negative bare quark mass, Aoki proposed that there may exist in the $g^{2}-M_{0}$ plane a phase in which one of three pions are condensed. Such a phase has been verified to exist in numerical simulations. Our first question is whether and where the Aoki phase exists in the case of the SU(2) gauge theories.

\section{Numerical study of Wilson-Dirac operator}

In this work we use two ensembles of quenched $\mathrm{SU}(2)$ gauge configurations on $8^{3} \times 16$ lattice generated with the Iwasaki gauge action at $\beta=0.8$ and 1.0. For each ensemble the HMC algorithm is used to generate 500 configurations each separated by 10 trajectories of unit length. The gauge field is represented by $2 \times 2$ link variable acting on the fermion spinors in the fundamental representation. For the adjoint fermions, the $3 \times 3$ link variable $U_{A}$ is obtained as

$$
\left(U_{A}\right)^{a b}=\frac{1}{2} \operatorname{tr}\left[T_{F}^{a} U T_{F}^{b} U^{\dagger}\right]
$$

where $T_{F}^{a}$ is the generator of the fundamental representation.

While there is no direct relation for the SU(2) gauge theory to the real world, just to give an idea of typical scale of these lattices, we set the lattice scale by hadronic radius $r_{0}$ determined from the static quark potential in fundamental representation. By setting $r_{0}=0.49 \mathrm{fm}$, the lattice spacings are determined as $a=0.2804(13) \mathrm{fm}$ at $\beta=0.8$ and $a=0.1445(15) \mathrm{fm}$ at $\beta=1.0$.

\subsection{Meson correlator}

As a probe of the Aoki phase, we first analyze the pion correlator following Ref. [4]. We introduce the twisted mass term as an external field in the Wilson-Dirac fermion action as

$$
S_{W t m}=\bar{\psi}\left[D_{W}-i m_{1} \tau_{3} \gamma_{5}\right] \psi
$$

Then in the Aoki phase, in the limit of $m_{1} \rightarrow 0, \pi_{3}$ becomes massive while $\pi_{ \pm}$remain massless. Thus we measure the pion correlator in the broken direction,

$$
\Gamma(x, y)=\left\langle\pi_{+}(x) \pi_{-}(y)\right\rangle, \quad \pi_{ \pm}(x)=i \bar{\psi}(x) \gamma_{5} \tau_{ \pm} \psi(x) .
$$

The extracted pion masses with standard fitting procedure are extrapolated to vanishing twisted mass. Except for the edges, the region where $m_{\pi}$ vanishes signals the Aoki phase.

Figure 1 shows the $m_{1}$ dependence of the pion mass at $\beta=1.0$. The left panel corresponds to the physical region (positive physical quark mass), while the right panel corresponds to the region in between the Aoki phase. As $M_{0}$ is changed, the $m_{1}$-dependence of the pion mass is changed differently in the left and right panels. 

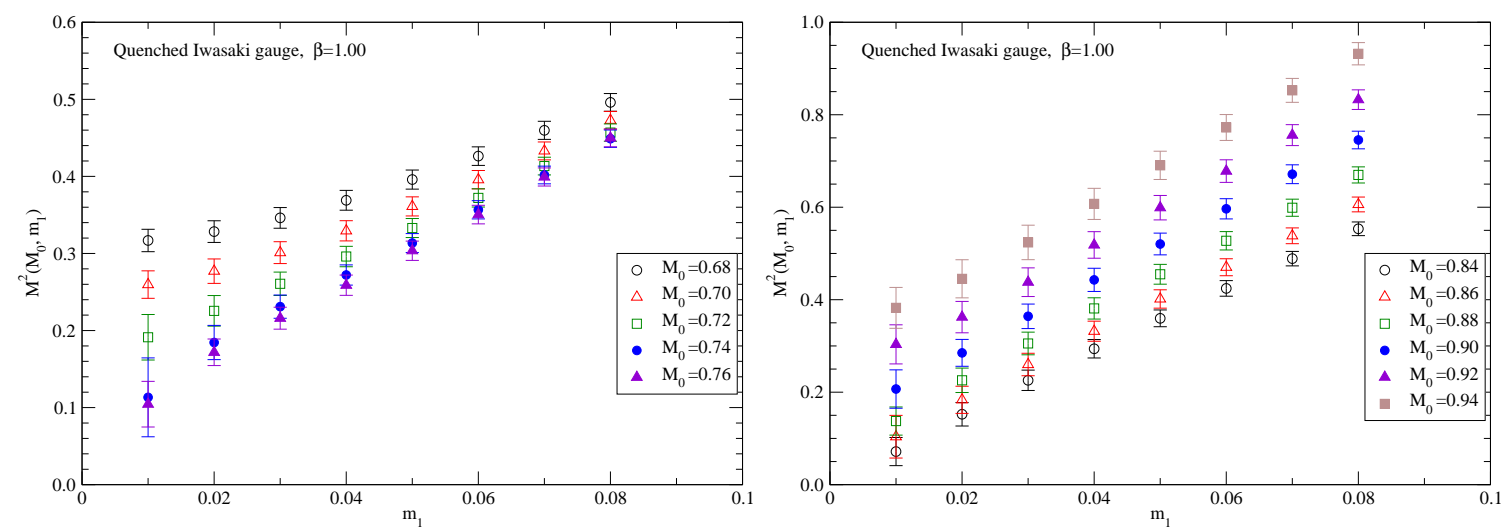

Figure 1: The $m_{1}$ dependence of the pion mass with the fundamental fermions at $\beta=1.0$. The left panel shows the result for $M_{0}$ in the physical region, and the right panel for $M_{0}$ in between the Aoki phase.
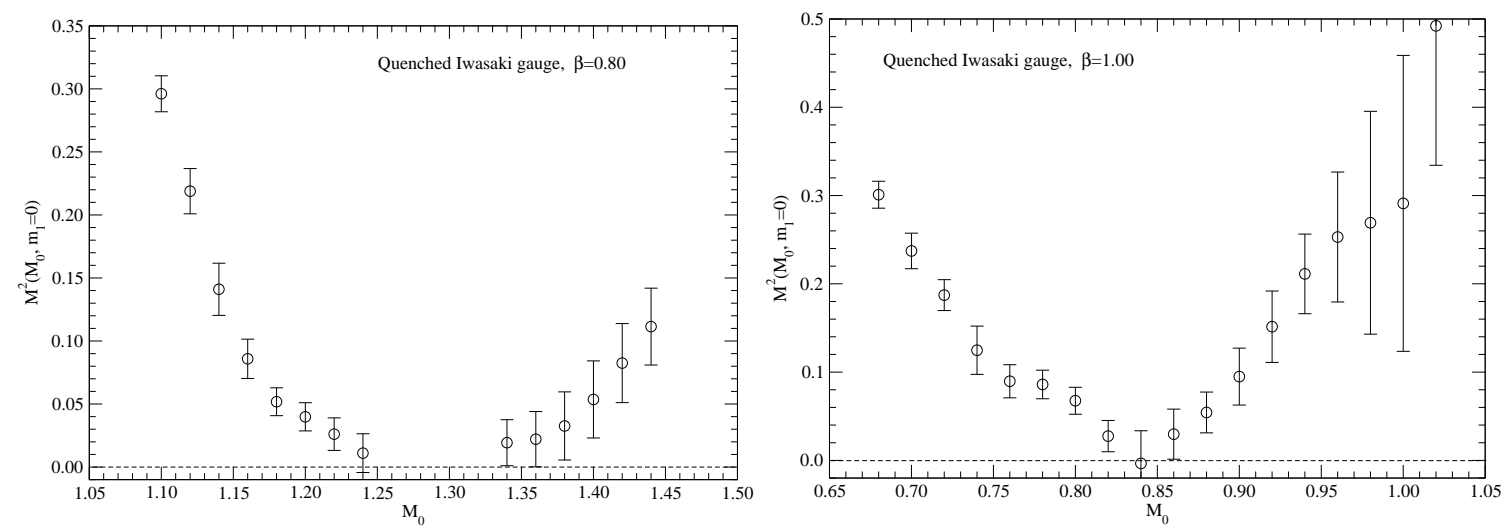

Figure 2: The values of pion mass at $m_{1}=0$ at $\beta=0.8$ (left panel) and 1.0 (right) for the fundamental fermions.

At each $M_{0}$, we linearly extrapolate the values of $m_{\pi}\left(m_{1}\right)^{2}$ to $m_{1}=0$ using the data at three lowest $m_{1}$. The result is plotted in Fig. 2. At $\beta=0.8$ (left panel), in the region of $M_{0}=1.24-1.35$ the pion mass is zero indicating that this region corresponds to the Aoki phase. In the right panel, at $\beta=1.0$, the region where the pion mass vanishes is very narrow. To examine whether this behavior depends on the volume, we are now repeating the same analysis on a $12^{3} \times 24$ lattice.

Figure 3 shows the result of the same analysis applied to the adjoint representation of fermions. At $\beta=0.8$ (left panel), in the wide range of $M_{0}>1.85$, the pion mass remains massless. This indicates that the Aoki phase extends over the whole region of $M_{0}>1.85$. At $\beta=1.0$ (right panel), however, finger-like structure appears.

\subsection{Low-lying eigenmodes of $H_{W}$}

In this subsection, we measure another probe of the Aoki phase, the low-lying eigenmodes of the hermitian Wilson-Dirac operator. In particular, if the low-lying spectrum has a gap, there is no possibility of the Aoki phase. Figure 4 shows the near-zero spectral density of $H_{W}$ at $\beta=1.0$. The left and right panel show the results at $M_{0}=0.82$ and 0.90, which are according to Fig. 2 very close to (or inside) the Aoki phase and outside the Aoki phase, respectively. In both cases, near- 

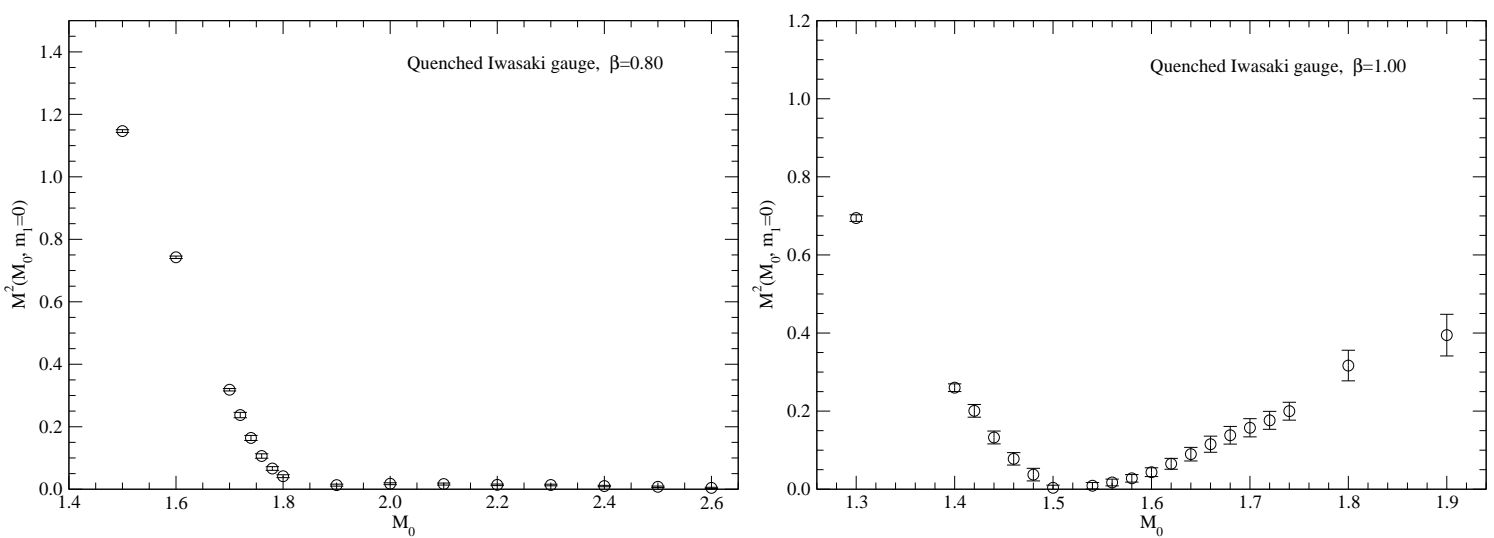

Figure 3: The values of pion mass at $m_{1}=0$ at $\beta=0.8$ and 1.0 for the adjoint fermions.
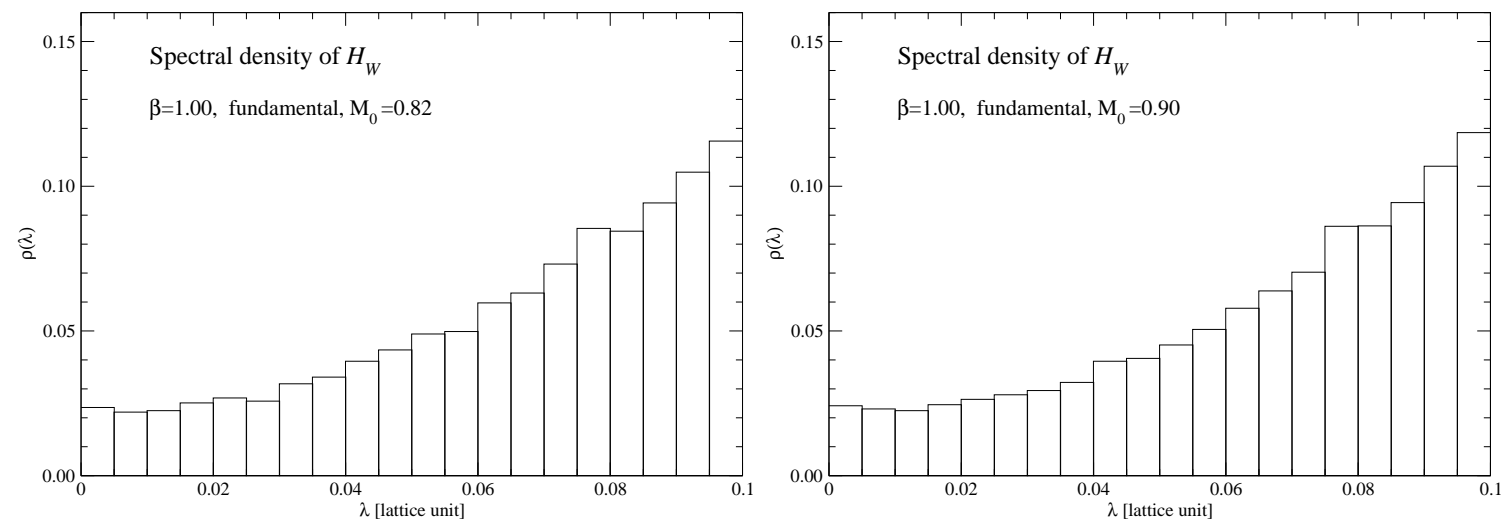

Figure 4: The low-lying spectral density of $H_{W}$ at $\beta=1.0$ for the fundamental fermions. Left and right panels show the results at $M_{0}=0.82$ and 0.90 , respectively.

zero spectral density is nonzero. This indicate that even outside Aoki phase, the spectral density is not necessarily to have a gap.

If the near-zero modes of $H_{W}$ have finite density, the properties of these modes are to be investigated. According to the conjecture of Golterman and Shamir, these modes should localize outside the Aoki phase, and extend inside. Figure 5 shows the behavior of the lowest mode on 5 configurations at the same values of $M_{0}$ as Fig. 4. These figures are generated as follows; first we find the site $x_{\max }$ at which the eigenvector $\phi_{\lambda}(x)$ has the largest value of $\left|\phi_{\lambda}(x)\right|^{2}$. Then along the $t$-direction, $\left|\phi_{\lambda}\left(x-x_{\max }\right)\right|^{2}$ is plotted. Difference between the results at $M_{0}=0.82$ and 0.90 is not manifest. More detailed analysis and comparison of more manifest cases are needed.

\section{Spectrum of overlap operator}

To investigate whether the chiral symmetry is broken or not, we determine the near-zero spectrum of the overlap-Dirac operator. Such analysis would be powerful tool to determine the chiral condensate in the $\varepsilon$-regime simulations. If the chiral symmetry is broken, the spectrum would exhibit specific feature as predicted by the random matrix theory (RMT) [10]. In the case of the 

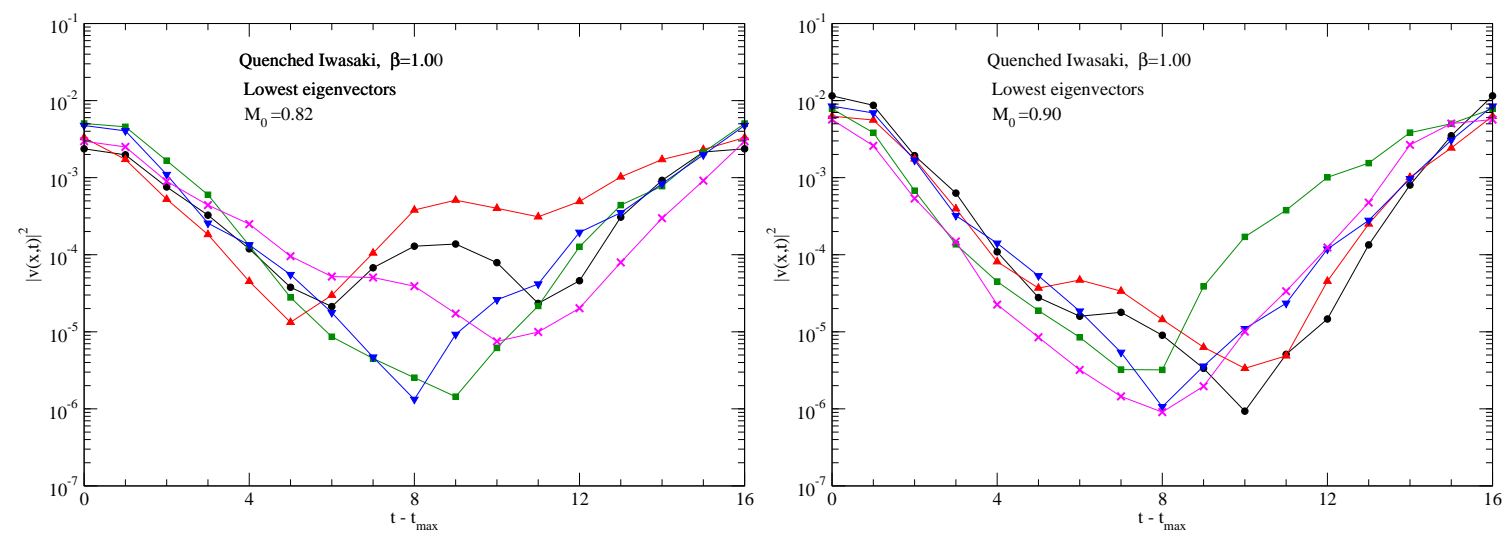

Figure 5: The behavior of the lowest eigenvector of $H_{W}$ at $\beta=1.0$ for the fundamental fermions. Left and right panels show the results at $M_{0}=0.82$ and 0.90 , respectively.
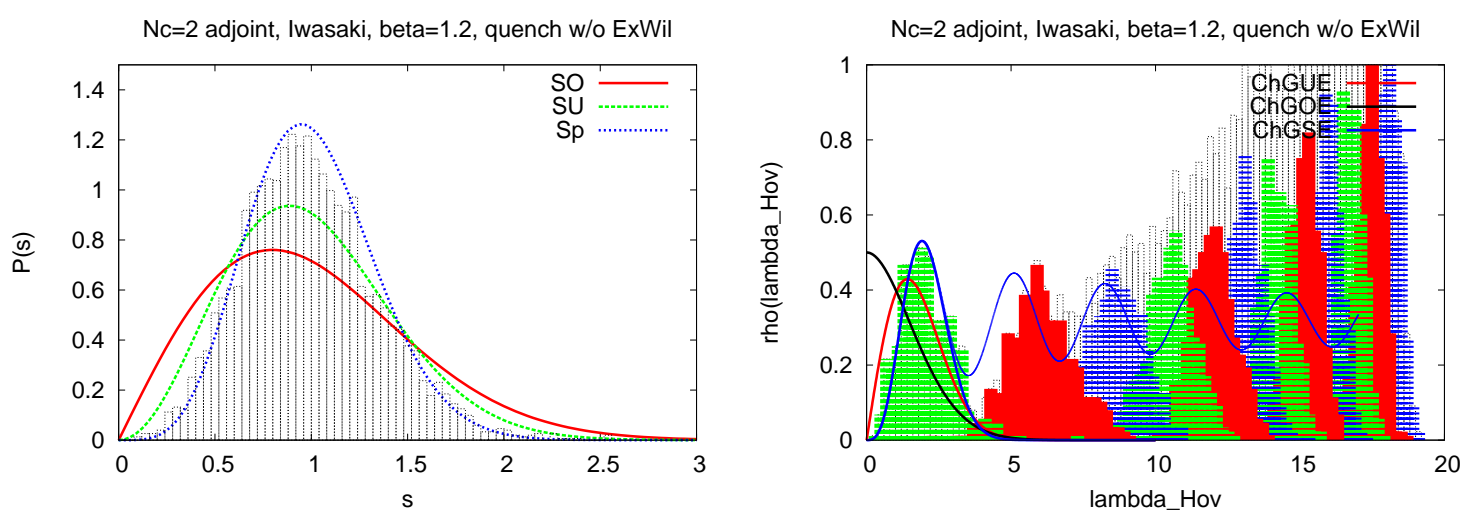

Figure 6: The result of the unfolded low-lying spectrum of the overlap operator. The left panel shows the distribution of the level spacing. The right panel compares the individual level distribution with three kinds of chiral RMT predictions. Each colored histogram displays th numerical result of each level. Solid lines are predictions of RMT for the lowest level. The dotted curve represents level distribution of ChGSE.

fundamental fermions in the color $\mathrm{SU}(2)$ gauge theory, the symmetry breaking pattern is expected to be $\mathrm{SU}\left(2 N_{f}\right) \rightarrow \mathrm{SO}\left(2 N_{f}\right)$, while for the adjoint fermions $\mathrm{SU}\left(2 N_{f}\right) \rightarrow \mathrm{Sp}\left(2 N_{f}\right)[9]$.

Figure 6 shows the result at $\beta=1.2$ for the adjoint representation $\left(M_{0}=1.8\right)$. Note that the lattice spacing at this $\beta$ is $0.066(2) \mathrm{fm}$ and thus volume is not sufficiently large. The left panel shows the distribution of unfolded level spacing. Three curves represent the predictions of RMT for the chiral Gaussian orthogonal, unitary, and symplectic ensembles. The result agrees with the symplectic ensemble. The right panel shows the distribution of unfolded eigenvalues. Three solid curves represent the predictions of RMT. The numerical result of the lowest level seems to agree with the chiral Gaussian symplectic ensemble (ChGSE). The distribution of the second lowest level, however, shifts rightward from the expected curve. This may be explained as the finite volume effect. These behavior indicate that the chiral symmetry is indeed broken with the expected pattern. 


\section{Conclusion and outlook}

As a preparation to the dynamical simulations of $S U(2)$ gauge theories with the overlap fermions, we have investigated the properties of the Wilson-Dirac operator on quenched ensembles. The meson correlators and eigenmodes of $H_{W}$ were analyzed to specify where the Aoki phase lies. These observables are indeed helpful to verify that the system is sound to use the overlap operator. We also observe the spectrum of the overlap operator to judge whether the chiral symmetry is broken or not.

Since the present analysis are still exploratory level, we need to complete them in wider range of $\beta$ and $M_{0}$, as well as on larger lattices. To improve the locality of the overlap operator, one may adopt the topology fixing term which explicitly suppresses the near-zero modes of $H_{W}[7,8]$. Fixed topology simulation in the $\varepsilon$-regime is also efficient for comparison with the chiral perturbation theory and RMT [11]. Since this is our plan in the dynamical overlap simulation, it is important to extend our analyses to this case.

We have started dynamical simulations with overlap fermions and a study of $N_{f}$-dependence of $\mathrm{SU}(2)$ gauge theories. It is also important to investigate the finite temperature phase transition of these theories.

Numerical simulations were performed on Hitachi SR11000 and IBM System Blue Gene Solution at High Energy Accelerator Research Organization (KEK) under a support of its Large Scale Simulation Program (No. 09-05). This work was supported in part by the Grant-in-Aid of the Ministry of Education (Nos. 19740160, 20105001, 20105002, 20105005, 21105503, 21540258).

\section{References}

[1] For recent review, G. T. Fleming, PoS LATTICE2008 (2008) 021 [arXiv:0812.2035 [hep-lat]]; E. Pallante, these proceedings.

[2] P. Hernandez, K. Jansen and M. Luscher, Nucl. Phys. B 552 (1999) 363 [arXiv:hep-lat/9808010].

[3] M. Golterman and Y. Shamir, Phys. Rev. D 68 (2003) 074501 [arXiv:hep-lat/0306002].

[4] M. Golterman, Y. Shamir and B. Svetitsky, Phys. Rev. D 71 (2005) 071502 [arXiv:hep-lat/0407021]; Phys. Rev. D 72 (2005) 034501 [arXiv:hep-lat/0503037].

[5] S. Aoki et al. [JLQCD Collaboration], Phys. Rev. D 78 (2008) 014508 [arXiv:0803.3197 [hep-lat]].

[6] S. Aoki, Phys. Rev. D 30 (1984) 2653.

[7] P. M. Vranas, arXiv:hep-lat/0001006; T. Izubuchi and C. Dawson [RBC Collaboration], Nucl. Phys. Proc. Suppl. 106 (2002) 748.

[8] H. Fukaya, S. Hashimoto, K. I. Ishikawa, T. Kaneko, H. Matsufuru, T. Onogi and N. Yamada [JLQCD Collaboration], Phys. Rev. D 74 (2006) 094505 [arXiv:hep-lat/0607020].

[9] M. E. Peskin, Nucl. Phys. B 175 (1980) 197.

[10] For a review, J. J. M. Verbaarschot and T. Wettig, Ann. Rev. Nucl. Part. Sci. 50 (2000) 343 [arXiv:hep-ph/0003017].

[11] H. Fukaya et al. [JLQCD Collaboration], Phys. Rev. Lett. 98 (2007) 172001 [arXiv:hep-lat/0702003]. 